\title{
TEREDINIDAE (MOLLUSCA, BIVALVIA) DO LITORAL DO PARAMA, BRASIL
}

\author{
Ana Claudia de Paula Mịller* \\ Paulo da Cunha Lana**
}

\begin{abstract}
A systematic survey of the Teredinidae (Mollusca, Bivalvia) of the Paraná coast (SE Brazil) is presented. The following species have been found, mainly in the mangrove areas: Lyrodus floridanus, Teredo navalis, Teredo bartschi, Teredo mindanensis, Neoteredo reynei, Nausitora fusticula, Bankia rochi, Bankia fimbriatula, Bankia bagidaensis, and Bankia gouldi. Keys, descriptions and figures of each species are included.

Key-words: Teredinidae; Taxonomy; Paraná (Brazil); wood-boring organisms.

\section{RESUMO}

Foi feito um levantamento sistemático das espécies de Teredinidae do estado do Paraná, na costa sudeste do Brasil. Coletas foram realizadas ao longo de todo o litoral paranaense. entre os anos de 1981 e 1984, com ênfase aos ambientes de manguezais, onde organismos perfuradores são mais abundantes. Foram identificadas as seguintes espécies: Lyrodus floridanus, Teredo navalis, Teredo bartschi, Teredo mindanensis, Neoteredo reynei, Nausitora fusticula, Bankia rochi, Bankia fimbriatula, Bankia bagidaensis, Bankia gouldi. Descrições, chaves

*IPARDES, Fundação Édison Vieira,

Rua Jayme Reis, 331

80.000 Curitiba, PR.

* CENTRO DE BIOLOGIA MARINHA, UFPR

Av. Beira Mar, s/n

83.200 Pontal do Sul, PR.
\end{abstract}


de identificação e desenhos foram elaborados para todas as espécies registradas.

PALAVRAS-CHAVE: Teredinidae; taxonomia; Paraná (Brasil); organismos perfuradores.

\section{INTRODUÇÃO}

Os organismos marinhos perfuradores de madeira constituem-se num permanente foco de interesse para o homem, em virtude dos enormes prejuízos causados a embarcações e instalações portuárias. A preocupação com os danos econômicos acarretados por suas atividades perfuradoras tem, no entanto, obscurecido o papel que eventualmente possam exercer nas cadeias alimentares detritívoras de ecossistemas estuarinos. É de máxima importância a atuação desses animais na recicla. gem da matéria orgânica de origem vegetal, notadamente em áreas de manguezais (TURNER, 1966; RAYNER, 1979; RIMMER et al., 1983).

A taxonomia dos bivalvos perfuradores da família Teredinidae, que conta com 66 espécies consideradas válidas, foi amplamente discutida e revista por TURNER (1966). Sua biologia foi extensivamente estudada por TURNER \& JOHNSON (1971). No entanto, são escassos os trabalhos que tratam da ocorrência, distribuição e biologia do grupo no Atlântico sulocidental. BASTIDA \& TORTI (1972) apresentaram dados sobre a distribuição de 17 espécies para a América do Sul. No Brasil, RIOS (1975) fêz referência a 6 gêneros e 14 espécies coletadas em placas experimentais instaladas da Bahia a São Paulo. BOFFI (1979), em seu trabalho sobre os moluscos brasileiros de interesse médico e econômico, citou cinco espécies de Teredinidae.

Em Pernambuco, FERNANDES \& COSTA (1967) estudaram à eficiência do uso de substâncias tóxicas preventivas em madeiras. Pesquisas sobre testes de resistência de diversos tipos de madeira ao ataque de Teredinidae foram realizadas no Rio Grande do Sul (STILNER, 1977) e Rio Grande do Norte (SERPA, 1978).

Recentemente, estudos sobre a ocorrência e aspectos da biologia desses perfuradores foram desenvolvidos em São Pau- 
Io (LAMPARELLI \& TIAGO, 1983), no Rio de Janeiro (SILVA et. al., 1980; SILVA et al., 1983) e no Paraná (MÜLLER, 1983).

A ausência de levantamentos sistemáticos detalhados dos perfuradores marinhos na costa brasileira tem dificultado a identificação destes organismos em trabalhos de controle e prevenção. No litoral paranaense o conhecimento destes organismos é de particular importância em virtude da existência de uma frota pesqueira de caráter predominantemente artesanal, composta de pequenas e médias embarcações de madeira. Tendo em vista os elevados prejuízos causados por estes perfuradores, informações referentes ao recrutamento, mecanismos de perfuração e resistência de madeiras são indispensáveis para o efetivo controle e monitoração de suas populações. Um embasamento sistemático seguro e consistente é imprescindível para a condução de estudos desta natureza.

O presente trabalho tem por objetivo realizar o levantamento sistemático das espécies de Teredinidae do litoral paranaense, fornecendo ilustrações e chaves de identificação que facilitem o reconhecimento destas formas em trabalhos de biologia e ecologia.

\section{MATERIAL E MÉTODOS}

\section{LOCAIS DE COLETA}

O trabalho foi desenvolvido em vários pontos do litoral paranaense, compreendendo praias arenosas, costões rochosos e os manguezais das baías de Paranaguá e Guaratuba. Os prin. cipais pontos de coleta, detalhados nos mapas 1 e 2, foram:

- manguezais do tipo Ribeirinho, na foz de rios: Guamandituba (ilha das Peças, baía de Paranaguá), Baguaçu (Pontal do Sul, Paranaguá), Maciel (baía de Paranaguá) e Penedo (Pontal do Sul, Paranaguá);

- manguezais dos tipos Franja e Ilhote: mar de Dentro (ilha do Mel, baía de Paranaguá), ponta sul e ponta norte da ilha das Peças (baía de Paranaguá), ilha do Veiga (baía de Guaratuba), ilha do Capinzal (baía de Guaratuba), ilha dos Papagaios (baía de Guaratuba), foz do rio do Cedro (baía de Guaratuba), foz do rio Boguaçu (baía de Guaratuba) e ilha da Sepultura (baía de Guaratuba); 
- manguezais pouco desenvolvidos, predominantemente de Laguncularia, nas margens das gamboas Penedo e Perequê (Pontal do Sul, Paranaguá);

- costões rochosos da praia Mansa (Caiobá) e praia da ponta do Joaquim (ilha do Mel);

- praias arenosas de Pontal do Sul e Anêmonas (Caiobá);

- plataforma continental (Estação 6122 da Operação Sueste I - convênio DHN/CBM-UFPR).

\section{METODOLOGIA E TRATAMENTO DO MATERIAL}

O material estudado foi obtido através de coleta manual de troncos soltos em decomposição ou com a utilização de coletores de pinho ou cedro, instalados em estações fixas nos manguezais da região.

Depois de retirados da madeira, os organismos coletados foram fixados em formol $10 \%$ e rotineiramente conservados em solução de álcool $75 \%$ e glicerina (5:1).

A identificação baseou-se principalmente nas monografias de TURNER (1966, 1971).

O material estudado encontra-se depositado, em parte, na coleção de referência do Centro de Biologia Marinha, Univers:dade Federal do Paraná (MCBM-BBI 01 a MCBM-BBI 79). Parte do material foi enviado para o Museum of Comparative Zoology, da Harvard University, para a confirmação das identificações.

\section{PARTE SISTEMATICA}

Chave para as espécies de Teredinidae registradas no litoral sudeste do Brasil.

1. Paletas com lâminas não segmentadas (fig. 6) ...... 2 Paletas com lâminas segmentadas (fig. 14); segmentos fundidos, porém vestigiais (fig. 13), ou claramente separados, formando cones (fig. 15) ............ 8 8

2. Lâmina com porção calcária pouco desenvolvida (fig. 1); periostraco marrom-escuro em forma de capa, muito desenvolvido, estendendo-se pronunciadamente além da lâmina (fig. 2) ..................... 3 
Lâmina com porção calcária muito desenvolvida (fig. 4); periostraco acompanhando o contorno da lâmina, não se estendendo muito além dela .............. 4

3. Animais com longo período de incubação, larvas liberadas no estágio pediveliger ......... Lyrodus pedicellatus * Animais com curto período de incubação, larvas liberadas no estágio charneira-reta (fig. 1) .. Lyrodus floridanus *

4. Paletas pequenas, frágeis, com formato variável (figs. 8-9) ....................... 5 Paletas largas, sólidas e fortemente calcificadas (fig. 4); pedúnculo curto e grosso; periostraco fino e claro; região posterior do animal com duas dobras carnosas (fig. 3) ........................ Neoteredo reynei

5. Periostraco fino, não se estendendo além da porção calcária (fig. 1) ..................... 6 Periostraco mais desenvolvido, estendendo-se além da porção calcária em margem estreita ou em pequenas aristas laterais (fig. 9) $\ldots \ldots \ldots \ldots \ldots \ldots \ldots \ldots \ldots, 7$

6. Lâmina com sulco mediano; margem distal da face externa em forma de " $V$ " profundo e margem da face inter.ıa em forma de " $U$ " menos acentuado .. Teredo furcifera"* Lâmina sem sulco mediano (fig. 6); margem distal da face externa em forma de " $U$ " não muito acentuado e margem distal da face interna levemente côncava (figs. 6-7); periostraco amarelo-palha, cobrindo a metade distal da lâmina (fig. 6) .................. Teredo navalis

7. Peristraco amarelo-ouro, cobrindo a metade distal da lâmina; margens distais da face interna e externa côncavas (fig. 9) ................... Teredo bartschi Periostraco marrom-escuro, mais desenvolvido, cobrindo toda a lâmina e estendendo-se além da metade inferior do pedúnculo (fig. 10); lâmina larga, oca e com alargamento no pedúnculo (fig. 11) ........... Teredo mindanensis

- Uma discussão pormenorizada das diferenças destas espécies geminadas no que se refere às suas estratégias de reprodução foi apresentada por CALLOWAY \& TURNER (1983).

**Espécies não registradas no presente levantamento, mas referidas para o litoral sudeste do Brasil. 
8. Segmentos fundidos (fig. 14) $\ldots \ldots \ldots \ldots \ldots \ldots .9$ Segmentos não fundidos, formando cones (fig. 15) . . 10

9. Segmentos fundidos e indistintos; lâmina ovalada; sifão exalante com dois tentáculos longos e numerosos tentáculos curtos ................ Nototeredo knoxi * Segmentos fundidos, mas distintos (fig. 14); comprimento da lâmina e pedúnculo equivalentes; incrustações calcárias na porção distal da lâmina, podendo estar ausentes em jovens ou por desgaste em adultos; sifão inalante com 12 tentáculos largos (figs. 12 e 14) .. Nausitora fusticula

10. Margem dos cones não serrilhada (fig. 15) ........ 11 Margem dos cones serrilhada (fig. 19) ......... 12

11. Aristas curtas e delgadas, ligadas na face interna de modo a formar uma capa larga (fig. 16); margem periostracal interna mais larga que a margem externa (fig. 15); cones em forma de tulipa, bem próximos uns dos outros ......

Bankia gouldi Aristas curtas e largas, margem periostracal estreita e de tamanho igual nas duas faces; cones em forma afunilada, bem espaçados uns dos outros ....... Bankia carinata *

12. Aristas longas e serrilhadas (fig. 19); margem periostracal larga; serrilhados da margem externa curtos, serrilhados da margem interna longos e agudos (fig. 20); bordo da porção calcária em forma de "V" profundo (fig. 21) .... ...................... Bankia fimbriatula Aristas curtas e serrilhadas (fig. 24); margem periostracal larga; serrilhados agudos e de tamanho equivalente nas duas margens; cones em forma de tulipa moderadamente espaçados; margem da porção calcária em forma de "U" (fig. 23) .................... Bankia rochi Aristas curtas e largas (fig. 25); margem periostracal estreita; serrilhados das duas margens curtos e de tamanhos equivalentes; porção calcária quase atingindo a base dos serrilhados (fig. 26) .......... Bankia bagidaensis

- Lyrodus floridanus (Bartsch, 1922)

(figs. 1-2)

* Espécies não registradas no presente levantamento, mas referidas para o litoral sudeste do Brasil. 
Teredo (Teredops) floridana Bartsch, 1922:28, est. 22., fig. 1 ; est. 33 , fig. 1.

Lyrodus pedicellatus Turner, 1966:116, est. 1, fig. B

Lyrodus floridanus; Turner, 1983:66, figs. 1-4.

DESCRIÇÃO. Paletas simples, com lâmina não segmentada. Porção calcária da lâmina pouco desenvolvida, com forma lanceolada (fig. 1) e coberta por forte capa periostracal. Perios traco variando do marrom-escuro ao quase negro, fortemente desenvolvido, assentado sobre a metade distal da porção calcária e estendendo-se muito além desta, em ampla margem (figs. 1-2). Expansões periostracais laterais em forma de dois cornos pequenos. Porção distal da capa periostracal com cavidade ausente em alguns exemplares, desenvolvida em outros, e muitas vezes preenchida por detritos e grãos de areia. Pedúnculo levemente curvado, de tamanho muito variável.

MEDIDAS DE DISPERSÃO (em $\mathrm{mm}$, para $\mathrm{n}=20$ ). Comprimento da lâmina $=0,59 \pm 0,21$; largura da lâmina $=0,34$ $\pm 0,099$; comprimento do pedúnculo $=0,59 \pm 0,20$.

DISTRIBUIÇÃO. Espécie de ampla distribuição em águas temperadas ou tropicais.

MATERIAL EXAMINADO. Praia Mansa - Caiobá: 1/11/83, 66 exs. MCBM-BBI 055. Há um registro genérico, com identificação específica duvidosa, na estação 6122 da Operação Sueste I, sobre a plataforma continental.

DISCUSSÃO. L. floridanus, descrito por BARTSCH (1922) para a Flórida, foi colocado na sinonímia do cosmopolita L. pedicellatus por diversos autores. Estudos recentes sobre a biologia reprodutiva do primeiro (CALLOWAY \& TURNER, 1983) mostraram que L. floridanus é perfeitamente distinto de L. pedicellatus, por apresentar curtos períodos de incubação larval, com a liberação de larvas no estágio de charneira-reta (straighthinge). É problemática a distinção destas duas espécies geminadas com base unicamente em características morfológicas. Exemplares de Lyrodus provenientes da praia Mansa em Caiobá foram identificados como L. floridanus por TURNER (in litt., 1984). Cerca de 30 exemplares coletados em troncos de madeira, a 47 metros de profundidade na estação 6122 da Operação Sueste 
I são imaturos, o que impossibilita uma diagnose específica segura. L. pedicellatus foi anteriormente referido para a costa brasileira por ROCH (1931), MOLL (1941), LANGE DE MORRETES (1949), BASTIDA \& TORTI (1972), RIOS (1975) e SILVA et al. (1983). Não se descarta a possibilidade de que parte destes registros corresponda, na realidade, a L. floridanus. A série de exemplares examinados apresentou ampla variabilidade intra-específica no que se refere ao formato das paletas. Indivíduos muito pequenos possuem paletas com forma quadrangular e periostraco pouco desenvolvido sem formar cornos laterais agudos. Já exemplares maiores possuem periostraco forte e desenvolvido, prolongando-se em cornos laterais longos (fig. 1). Ainda em adultos, a cavidade distal do periostraco, solidificada em maior ou menor grau, pode apresentar-se vazia ou preenchida por detritos, o que confere formato de ampulheta à porção calcária da lâmina. L. floridanus foi freqüentemente encontrado associado a Bankia gouldi na região estudada.

- Neoteredo reynei (Bartsch, 1920)

(figs. 3-5)

Terodo (Neoteredo) reynei Bartsch, 1920:69-70; Bartsch, 1922:30, est. 23, est. 33 , fig. 3.

Neoteredo reynei; Turner, 1966:119, est. 32, fig. C.

DESCRIÇÃO. Paletas sólidas, não segmentadas e simétricas. Lâmina com porção calcária sólida não segmentada e em forma de pá (figs. 4-5). Face externa convexa e com leve depressão na extremidade distal (fig. 4); face interna côncava e lisa (fig. 5). Pedúnculo largo e não muito longo (fig. 4). Periostraco fino, castanho, cobrindo a metade superior distal da face externa da lâmina e podendo estar ausente ou vestigial em paletas desgastadas. Sifões unidos em todo o seu conıprimento, exceto na extremidade distal, com densa pigmentação marrom. Sifão exalante com bordo interno margeado por numerosas papilas. Sifão inalante menor. Manto muito desenvolvido, com duas dobras carnosas na porção posterior (fig. 3). Ceco muito desenvolvido, longo, largo e ventral, em geral apresenta-se muito distendido, com grande quantidade de detritos. 
MEDIDAS DE DISPERSÃO (em $\mathrm{mm}$, para $\mathrm{n}=20$ ). Comprimento da lâmina $=3,97 \pm 1,70$; largura da lâmina $=3,87$ $\pm 1,52$; comprimento do pedúnculo $=4,75 \pm 2,40$.

DISTRIBUIÇÃO. Costa oeste do Atlântico, em águas estuarinas tropicais e subtropicais; costa leste da Africa, de Serra Leoa ao Congo. No Brasil: São Paulo, Paraná.

MATERIAL EXAMINADO. Rio Penedo: 4/4/83, 4 exs. (MCBM-BBI 01); 6/3/83, 8 exs. (MCBM-BBI 02); rio Baguaçu; 17/11/83, 4 exs. (MCBM-BBI 03); 18/8/83, 3 exs. (MCBM-BBI 04); 19/10/83, 3 exs.; rio Guamandituba: 15/10/82, 1 ex. (МСBMBBI 06); 15/9/82, 1 ex. (MCBM-BBI 07); 26/1/82, 4 exs. (MCBMBBI 08); 18/10/82, 1 ex. (MCBM-BBI 09); 12/11/81, 10 exs. (MCBM-BBI 10); 29/9/81, 1 ex. (MCBM-BBI 05); rio Perequê: 17/8/83, 2 exs. (MCBM-BBI 11); rio Maciel: 20/1/84, 1 ex. (MCBM-BBI 12); Ponta Norte da llha das Peças: 13/12/83, 11 exs. (MCBM-BBI 13); Ponta Sul da llha das Peças: 13/12/83, 3 exs. (MCBM-BBI 14); baía de Guaratuba - ilha do Veiga: 18/1/84, 1 ex. (MCBM-BBI 64); ilha dos Papagaios: 30/1/84, 7 exs. (MCBM-BBI 65); foz do rio Cedro: $30 / 1 / 84,5$ exs. (MCBMBBI 66).

DISCUSSÃO. A espécie é facilmente diagnosticada pela presença de um par de dobras carnosas na região posterior e pela presença de um manto muito desenvolvido. A coloração do periostraco, quando presente, varia muito, do castanho-claro ao marrom-escuro. O tamanho dos indivíduos é variável, porém a espécie se caracteriza por atingir grande comprimento (210-500mm na coleção estudada). A espécie é abundante nos manguezais do litoral paranaense, predominando na baía de Paranaguá. Nos troncos de madeira cavam galerias largas e muito compridas, no sentido das fibras, localizadas geralmente muito abaixo do córtex e próximo da medula. Estas galerias são revestidas por forte lineamento calcário. N. reynei foi coletado em abundância em troncos em manguezais, não sendo, entretanto, observado qualquer recrutamento desta espécie nas placas instaladas nos mangues. $\mathbf{N}$. reynei foi comumente encontrado sozinho em troncos ou associado a Bankia fimbriatula.

- Teredo navalis Linnaeus, 1758 (figs. 6-7) 
Teredo navalis Linnaeus, 1758:657; Turner, 1966-112-113, est. 14, figs. A-E.

Teredo (Teredo) beachi Bartsch, 1921::29.

Teredo (Teredo) beaufortana Bartsch, 1922:22, est. 32, fig. 1

Teredo (Teredo) morsei Bartsch, 1922:21.

Teredo (Teredo) novanglia Bartsch, 1922:19, est. 21, fig. 3.

Teredo navalis borealis Roch, 1931:27, fig. 8.

DESCRIÇÃO. Paletas simples e não segmentadas. Lâmina com porção calcária sólida, em forma típica de pá, com face interna plana (fig. 7) e face externa convexa (fig. 6). Margem interna da porção calcária levemente côncava em forma de "U" (fig. 7) e margem externa mais cavada, também em forma de "U" (fig. 6). Periostraco fino, translúcido e amarelo-pálido, cobrindo a metade distal da face externa da lâmina e prolongando-se em margem estreita nas duas faces e lateralmente em cornos curtos (fig. 6-7). Pedúnculo curto, sólido e levemente curvado (fig. 6).

MEDIDAS DE DISPERSÃO (em $\mathrm{mm}$, para $\mathrm{n}=13$ ). Comprimento da lâmina $=1,47 \pm 0,63$; largura da lâmina $=0,98 \pm 0,35$; comprimento do pedúnculo $=0,90 \pm 0,54$.

DISTRIBUIÇÃO. Cosmopolita. No litoral do Brasil: Paraná e Rio Grande do Sul.

MATERIAL EXAMINADO. Caiobá - praia Mansa: 1/11/83, 8 exs. (MCBM-BBI 29); ponta sul da ilha das Peças: $13 / 12 / 83$, 1 ex. (MCBM-BBI 67); ilha do Mel - ponta do Joaquim: 2/11/83, 1 ex. (MCBM-BBI 68); rio Baguaçu: 14/2/83, 1 ex. (MCBM-BB! 030).

DISCUSSÃO. T. navalis diferencia-se de T. bartschi, que apresenta periostraco amarelo-ouro e lâmina mais larga, com formato quadrangular. No litoral paranaense os exemplares apresentaram sempre um periostraco amarelo-palha e fino, ausente em alguns exemplares mais desenvolvidos. A ampla va riabilidade intra-específica em $\mathbf{T}$. navalis tem gerado dificuldades para sua correta caracterização, fato este evidenciado por BARTSCH (1922), que baseando-se apenas na morfologia das paletas descreveu quatro novas espécies. Segundo MILLER 
(1923), variações na largura da lâmina, tamanho do pedúnculo e cor do periostraco podem ser determinadas por fatores ecclógicos, pelo tipo de madeira e pela idade dos indivíduos. T. navalis foi referido para o litoral sul-brasileiro por RIOS (1975). T. furcifera, espécie circum-tropical semelhante a $T$. navalis, foi registrado próximo à área de estudo na localidade de São Francisco do Sul (ROCH \& MOLL, 1935), sob o nome de T. krappei. T. furcifera distingue-se de $\mathbf{T}$. navalis por apresentar um sulco transversal na porção mais larga da lâmina.

- Teredo bartschi Clapp, 1923:33

(figs. 30-31)

Teredo bartschi Clapp, 1923:33, est-3-4; Turner, 1966:90, est. 8, figs. A-F.

Teredo (Teredo) batilliformes Clapp, 1924-282, est. 1, figs. 1-6; est. 3, figs. 13-14.

Teredo (Teredo) hiloensis Edmondson, 1942:113, figs. 4d-h.

DESCRIÇÃO. Paletas simples. Lâmina inteira e não segmentada em cones (fig. 8). Porção calcária da lâmina com face interna plana e face externa ligeiramente côncava; porção calcária não se estendendo até o topo da lâmina (fig. 9). Periostraco fino, amarelo palha, envolvendo a metade distal da lâmina e projetando-se em pequenas aristas laterais. Margem periostracal da face interna e externa em forma de "U". Pedúnculo de comprimento em geral menor que 0 da lâmina.

MEDIDAS DE UM EXEMPLAR (em $\mathrm{mm}$ ). Comprimento da lâmina $=1,6$; largura da lâmina $=0,7$; comprimento do pedúnculo $=1,0$.

DISTRIBUIÇÃO. Cosmopolita: tropical e subtropical. No Brasil: Bahia, São Paulo e Paraná.

MATERIAL EXAMINADO. Caiobá - praia Mansa: 1/11/83, 5 exs. (MCBM-BBI 50).

DISCUSSÃO. A espécie T. bartschi assemelha-se muito a T. navalis no formato e tamanho da lâmina, sendo que em $\mathbf{T}$. navalis o periostraco cobre toda a porção calcária, não se estendendo além desta. EDMONDSON (1942), em sua descrição de $T$. bartschi, assinala como característica importante o fato 
de nesta espécie o comprimento da lâmina ser menor que o comprimento do pedúnculo. Entretanto, ainda no mesmo trabalho, cita como espécie nova T. hiloensis (atualmente T. bartschi), distinguindo-o de T. bartschi pelo formato da lâmina, cor do periostraco e comprimento da lâmina maior que o do pedúnculo. Nos exemplares observados, o periostraco apresentou-se sempre de cor amarelo-palha. Consideramos a cor do periostraco caráter insuficiente para distinção das espécies acima referidas, já que TURNER (1971) atribui a T. bartschi am. pla variabilidade na cor do periostraco, que vai do ouro ao marrom-escuro. Todos os exemplares coletados são muito pequenos e provavelmente imaturos.

- Teredo mindanensis Bartsch, 1923

(figs. 32-33)

Teredo (Coleoteredo) mindanensis Bartsch, 1923:99; Bartsch, 1927:539, est. 53, figs. 10, 12 ; est. 56 , fig. 6 ; est. 60 , figs. 4, 8, 12; Turner, 1966:111, est. 12, figs. D e E.

Teredo (Coleoteredo) bayeri Roch, 1955:130, figs. 4a-g.

DESCRIÇÃO. Paleta sólida e simples. Lâmina não segmentada em cones, com formato quadrangular e oca até o pedúnculo; face interna plana e face externa côncava (fig. 10). Margem da face externa da porção calcária em forma de " $U$ " e da face interna em forma de "V" não muito acentuada (figs. 10-11). Periostraco grosso, marrom-escuro, cobrindo toda a porção calcária da lâmina, estendendo-se além desta em margem estreita (fig. 10). Periostraco estendendo-se também para baixo cobrindo a metade anterior do pedúnculo. Pedúnculo sólido, curto, levemente curvado e claramente alargado na sua parte mediana (fig. 10).

MEDIDAS DE DISPERSÃO (em $\mathrm{mm}$, para $\mathrm{n}=3$ ). Comprimento da lâmina $=1,43 \pm 0,58$; largura da lâmina $=1,16 \pm$ 0,28 ; comprimento do pedúnculo $=0,83 \pm 0,35$.

DISTRIBUIÇÃO. Nova Guiné, Java, Austrália tropical. Brasil: Paraná.

MATERIAL EXAMINADO. Rio Baguaçu: 5/4/83, 5 exs. (MCBM-BBI 52); baía de Guaratuba - ilha do Veiga: 30/1/84, 1 ex. (MCBM-BBI 69). 
DISCUSSÃO. TURNER, em sua chave para as espécies do grupo (1971), atribui a T. mindanensis lâmina de formato triangular e periostraco fino e amarelo. Os exemplares paranaenses, apesar de apresentarem a lâmina oca e a pequena saliência no pedúnculo diagnósticas da espécie, possuem lâmina quadrangular e periostraco grosso marrom-escuro. A identificação específica foi confirmada pela própria TURNER (in litt., 1984). Não se descarta, no entanto, a possibilidade de que estes exemplares representem espécie nova, fato que só poderá ser estabelecido com coletas mais intensas e comparação com material tipo.

- Nausitora fusticula (Jeffreys, 1860)

(figs. 12-14)

Toredo fusticulus Jeffreys, 1860:125.

Bankia (Nausitora) brasiliensis Bartsch, 1922:15, est. 20, fig. 3; est. 31, fig. 1.

Bankia (Nausitora) excolpa Bartsch, 1922:13, est. 8, fig. 2; est. 31, fig. 4.

Nausitora fusticula (Jeffreys). Turner, 1966:102, est. 37, fig. B.

DESCRIÇÃO. Paletas sólidas, bem desenvolvidas e assimétricas (figs. 12, 13). Lâmina com segmentos distintos, porém fundidos (figs. 12-13). Face externa da lâmina coberta por periostraco grosso, de coloração variando do amarelo ao marrom, com inscrustações papilosas na extremidade distal (figs. 12, 14); face interna com metade inferior lisa e contínua e metade superior composta de segmentos calcários paralelos ou ligeiramente oblíquos, intercalados por camadas de periostraco que se prolongam lateralmente (figs. 13-14). Pedúnculo sólido e alongado, com eixo reto ou levemente torcido (figs. 12-13). Sifões com pigmentação marrom intensa. Sifão inalante com 12 tentáculos terminais longos, agrupados dois a dois, formando seis lóbulos distintos (fig. 14). Sifão exalante liso, sem tentáculos.

MEDIDAS DE DISPERSÃO (em $\mathrm{mm}$, para $\mathrm{n}=20$ ). Comprimento da lâmina $=4,52 \pm 1,44$; largura da lâmina $=3,14 \pm$ 1,00; comprimento do pedúnculo $=6,65 \pm 2,31$. 
DISTRIBUIÇÃO. Atlântico ocidental (Brasil, Uruguai); Pacífico oriental (Peru). No litoral brasileiro: São Paulo e Paraná.

MATERIAL EXAMINADO. Rio Baguaçu: 18/8/83, 16 exs. (MCBM-BBI 15); 5/4/83, 1 ex. (MCBM-BBI 18); 17/11/83, 2 exs. (MCBM-BBI 19); 19/10/83, 20 exs. (MCBM-BBI 26); rio Penedo: 6/3/83, 6 exs. (MCBM-BBI 16); 4/4/83, 2 exs. (МCBM-BBI 17); rio Guamandituba: 29/9/81, 4 exs. (MCBM-BBI 20); 15/9/82, 1 ex. (MCBM-BBI 21); 26/1/82, 15 exs. (MCBM-BBI 22); ilha do Mel: $2 / 11 / 83,13$ exs. (MCBM-BBI 23); ponta norte da ilha das Peças: $13 / 12 / 83,34$ exs. (MCBM-BBI 26); ponta sul da ilha das Peças: $13 / 12 / 83,9$ exs. (MCBM-BBI 25); baía de Guaratuba ilha do Veiga: $18 / 1 / 84,6$ exs. (MCBM-BBI 70); ilha do Capinzal: $18 / 1 / 84,17$ exs. (MCBM-BBI 71 ); foz do rio Cedro: $30 / 1 / 84$, 6 exs. (MCBM-BBI 72); ilha dos Papagaios: 30/1/84, 18 exs. (MCBM-BBI 73); foz do rio Boguaçu: 18/1/84, 25 exs. (MCBMBBI 74).

DISCUSSÃO. A espécie é facilmente diagnosticada pela presença de sifão inalante com 12 tentáculos longos, agrupados em lóbulos distintos. Diferencia-se das outras espécies do gênero por possuir o pedúnculo das paletas longo e bem maior que a lâmina. As inscrustações calcárias papilosas podem, muitas vezes, estar ausentes em indivíduos jovens ou, por desgaste, em adultos (fig. 12). Espécie restrita aos manguezais, predominante na baía de Guaratuba e abundante, juntamente com Neoteredo reynei, na baía de Paranaguá. A espécie foi anteriormente referida para o litoral sul do Brasil por BARTSCH (1922) e RIOS (1975).

- Bankia gouldi (Bartsch, 1908)

(figs. 43-46)

Xylotra gouldi Bartsch, 1908:211.

Bankia (Bankiella) mexicana Bartsch, 1921:10, fig. 2; Bartsch, 1922:10, fig. 2.

Bankia schrencki Moll, 1935:275, est. 2, fig. 7.

Bankia gouldi (Bartsch). Clench \& Turner, 1946:13-15, est. 9, figs. 1-4; Turner, 1966:103, ests. 59 e $60 \mathrm{~F}$.

DESCRIÇÃO. Conchas típicas da família (figs. 45-46). Paletas com simetria bilateral. Lâmina composta por vários seg- 
mentos em forma de cones afunilados, não fundidos e muito próximos uns dos outros (fig. 15). Margem interna da porção calcária de cada segmento em forma de " $U$ " mais profunda que a margem externa, também em forma de " $U$ ", mais larga e não tão cavada (figs. 15-16). Periostraco translúcido e amarelado, cobrindo toda a porção calcária e estendendo-se lateralmente além desta em cornos curtos e finos. Bordo periostracal das faces externa e interna liso (fig. 16). Margem periostracal externa estreita e margem interna mais larga formando uma tela que une as aristas laterais (figs. 15-16). Pedúnculo delgado e longo (fig. 15).

MEDIDAS DE DISPERSÃO (em $\mathrm{mm}$, para $\mathrm{n}=19$ ). Comprimento da lâmina $=3,67 \pm 1,34 ;$ largura da lâmina $=1,34$ $\pm 0,33$; comprimento do pedúnculo $=2,05 \pm 0,71$.

DISTRIBUIC̣ÃO. Atlântico ocidental. em áquas tropicais e temperadas: New Jersey, Antilhas, Venezuela, Uruguai e Brasil; costa pacífica do Panamá. No litoral do Brasil: Bahia, Rio de Janeiro, São Paulo, Paraná e Santa Catarina.

MATERIAL EXAMINADO. Caiobá - praia Mansa: 1/11/83, 110 exs. (MCBM-BBI 27); ponta sul da ilha das Peças: 13/12/83, 6 exs. (MCBM-BBI 28).

DISCUSSÃO. Espécie amplamente distribuída, sendo a mais abundante do gênero Bankia na costa atlântica. É considerada também a mais destrutiva nestas águas (BARTSCH, 1922; CLENCH \& TURNER, 1946). Foi encontrada na costa oeste da América Central e classificada como B. mexicana por BARTSCH (1922). Entretanto, o exame do tipo por CLENCH \& TURNER (1946) revelou que os caracteres usados por BARTSCH (1922) para diferenciar B. gouldi de B. mexicana correspondiam a variações intra-específicas de B. gouldi. Moll (in CLENCH \& TURNER, 1946) descreveu como B. schrencki exemplares co letados em São Francisco do Sul, Santa Catarina. Os espécimes descritos estavam secos e com periostraco vestigial. Baseando-se nestes espécimes mal preservados, Moll descreveu os segmentos como dotados de uma membrana larga e com franja curta. $\mathrm{Na}$ realidade, o periostraco quando seco ou mal preservado produz serrilhados artificiais (CLENCH \& TURNER, 1946). No entanto, alguns exemplares do litoral paranaense apresentaram a margem periostracal levemente serrilhada, lan- 
çando dúvidas quanto à identificação. Esta variação, provavelmente de natureza intra-específica, ocorreu em certos exemplares de um grande lote de organismos provenientes de troncos coletados entre pedras, durante a baixa-mar, na praia Mansa, em Caiobá. B. gouldi é espécie próxima de B. carinata, cosmopolita, que possui margem periostracal também lisa e porção calcária em forma de funil. B. carinata apresenta, no entanto, cornos laterais longos e cones embriônicos compactos e cobertos por capa periostracal. B. gouldi é característica do litoral oceânico da costa sudeste brasileira (LAMPARELLI \& TIAGO, 1983; SILVA et al., 1983). No presente trabalho foi registrada, pela primeira vez, em manguezal próximo à barra de acesso à baía de Paranaguá.

- Bankia fimbriatula Moll \& Roch, 1931

(figs. 17-20)

Teredo palmulata Forbes \& Hanley, 1835:86, est. 2, figs. 9-11, non palmulata Lamarck, 1818.

Teredo fimbriata Jeffreys, 1860:126, non fimbriata Defrance, 1828, non philippi 1836.

Bankia fimbriatula Moll \& Roch, 1931:213, est. 25, fig. 37, new name for fimbriata Jeffreys, 1860; Clench \& Turner, 1946: 22-23, est. 14, figs. 1-4; Turner, 1966: 101, ests. 55-56, fig. a.

Bankia canalis Bartsch, 1944:1, est. 1.

DESCRIÇÃO. Paletas compridas e delicadas. Lâmina com certa de 10-15 segmentos, separados em cones distintos (fig. 17), em forma de "V", moderadamente espaçados nos exemplares mais desenvolvidos e bem espaçados em exemplares jovens. Cones calcários com expansões laterais de comprimento desigual (fig. 19). Periostraco com coloração variando de amarelo-pálido nos exemplares menores a amarelo-ouro nos exemplares mais desenvolvidos, estendendo-se bem adiante da porção calcária e formando margem larga e serrilhada (figs. 18-19). Margens interna e externa cavadas em forma de "U" profundo (figs. 19-20). Bordo da face interna com serrilhados finos e longos (fig. 20); bordo da face externa com serrilhados curtos e menos agudos (fig. 19). Cornos laterais, longos, agu- 
dos e serrilhados (fig. 20), muitas vezes duplos, sendo um mais curto que o outro. Pedúnculo fino e longo.

MEDIDAS DE DISPERSÃO (em $\mathrm{mm}$, para $\mathrm{n}=16$ ). Comprimento da lâmina $=5,43 \pm 3,78$; largura da lâmina $=1,16 \pm 0,46$; comprimento do pedúnculo $=4,47 \pm 2,17$.

DISTRIBUIÇÃO. Espécie de distribuição anfi-atlântica (costa leste e oeste da Flórida, Antilhas, costa leste da América Central e América do Sul, costa atlântica da Europa). Costa pacífica do Panamá. No Brasil: Bahia, São Paulo e Paraná.

MATERIAL EXAMINADO. Rio Guamandituba: 10/5/82, 5 exs. (MCBM-BBI 31); 12/11/81, 2 exs. (MCBM-BBI 32); 13/7/82, 26 exs. (MCBM-BBI 33); placa 5A: 24/11/82, 20 exs. (MCBM-BBI 34); rio Baguaçu: 18/8/83, 2 exs. (MCBM-BBI 49); baía de Paranaguá: sem data, 1 ex. (MCBM-BBI 35).

DISCUSSÃO. A espécie caracteriza-se pela presença de margem periostracal larga e serrilhada e cornos laterais longos e agudos. Distingue-se de B. australis por possuir serrilhados de tamanho desigual; estes serrilhados podem apresentar-se duplicados em B. australis. Alguns exemplares apresentaram par$t \in$ ou a totalidade das paletas cobertas por uma prolongação do manto e sifões muito longos e distendidos, ultrapassando muitas vezes o comprimento das paletas. A maior incidência desta espécie foi registrada em troncos de Laguncularia racemosa e, secundariamente, em troncos de Rhizophora mangle. Nos troncos cavam galerias, preferencialmente na região do córtex, dispostas transversalmente ao sentido das fibras. Os alinhamentos calcários nas regiões terminais apresentam-se tipicamente arranjados em grupos cônicos.

- Bankia rochi Moll, 1931

(figs. 17-24)

Bankia rochi Moll, 1931:215, est. 25; Turner, 1966:119, est. 56, fig. C.

Bankia (Neobankia) roonwali Rajagopalaiengar, 1961:550.

DESCRIÇÃO. Paletas formadas por lâmina com segmentos separados, bem próximos uns dos outros (fig. 17), em forma de tulipa. Margens interna e externa da porção calcária em 
forma de " $U$ ", sendo a curvatura da margem interna mais acentuada (profunda) que a da margem externa (figs. 23-24). Periostraco amarelo-ouro a marrom-avermelhado, recobrindo toda a porção calcária de cada cone e estendendo-se além desta em margem larga e serrilhada (figs. 22-23). Bordos das faces interna e externa com serrilhados quase iguais em tamanho e forma aguda. Os serrilhados da margem externa são, algumas vezes, menores que os serrilhados da margem interna (figs. 23-24). Cornos laterais curtos e serrilhados, pouco afinados nas extremidades e acompanhados por uma bractea periostracal (figs. 21 e 24).

MEDIDAS DE DISPERSÃO (em $\mathrm{mm}$, para $\mathrm{n}=20$ ). Comprimento da lâmina $=4,97 \pm 1,67$; largura da lâmina $=1,60 \pm$ 0,42 ; comprimento do pedúnculo $=4,50 \pm 1,47$.

DISTRIBUIÇÃO. Indo-Pacífico, em áreas tropicais e subtropicais.

MATERIAL EXAMINADO. Rio Baguaçu: 5/4/83, 2 exs. (MCBM-BBI 37); 18/8/83, 12 exs. (MCBM-BBI 40); 19/10/83, 13 exs. (MCBM-BBI 42); rio Guamandituba: 12/11/81, 20 exs. MCBM-BBI 36); 13/8/82, 11 exs. (MCBM-BBI 38); sem data, 15 exs. (MCBM-BBI 39); 10/5/82, 48 exs. (MCBM-BBI 43); rio Penedo: 4/4/83, 1 ex. (MCBM-BBI 47); 6/3/83, 1 ex. (MCBM-BBI 44); ponta norte da ilha das Peças: $13 / 12 / 83,6$ exs. (MCBM-BBI 45); fonta sul da ilha das Peças: $13 / 1 / 83,9$ exs. (MCBM-BBI 75); baía de Guaratuba - ilha do Capinzal: 30/1/84, 20 exs. (MCBM-BBI 76); ilha do Veiga: 18/1/84, 19 exs. (MCBM-BBI 77); foz do rio Cedro: 30/1/84, 26 exs. (MCBM-BBI 78); ilha dos Papagaios: 18/1/84, 10 exs. MCBM-BBI 79).

DISCUSSÃO. B. rochi, espécie indo-pacifica possivelmente introduzida no Brasil através de navios de madeira, é facilmente identificada pela presença de acúleos periostracais, que saem da base de cada segmento e se estendem junto aos cornos laterais. Estes acúleos muitas vezes apresentam-se aderidos à capa periostracal dos cones, sendo de difícil observação. Segundo a chave de identificação de Turner (1971-55) os cornos de B. rochi são lisos, sendo que Turner (1966:74) ilustra a espécie com cornos levemente serrilhados. As espécies observadas no litoral paranaense possuem cornos com serrilhados não agudos, porém evidentes. Em alguns exemplares obser- 
vou-se que os serrilhados da margem periostracal podem, muitas vezes, estar bifurcados (divididos). (figs. 23 e 24). Apesar desta característica ser citada como específica de B. australis (TURNER, 1966, 1971), acredita-se ser esta uma variação intraespecífica de B. rochi.

- Bankia bagidaensis Roch, 1929

(figs. 55-57)

Bankia bagidaensis Roch, 1929:18, est. 2, fig. 17; Turner, 1966:89, est. 51, fig. D.

DESCRIÇÃO. Paletas assimétricas. Lâmina com segmenttos moderadamente separados uns dos outros (figs. 26-27). Margem interna e externa da porção calcária com forma côncava, pouco acentuada (fig. 25). Periostraco variando de amarelopardo a marrom-avermelhado, recobrindo toda a porção calcária de cada cone e estendendo-se além desta em margem estreita e serrilhada (fig. 25). Porção calcária quase atingindo a base dos serrilhados (fig. 25). Serrilhados das margens externa e interna de tamanho equivalente. Cornos laterais largos e serrilhados, de comprimento variável, desde curtos a longos (figs. 25-26).

MEDIDAS DE DISPERSÃO (em $\mathrm{mm}$, para $\mathrm{n}=16)$. Comprimento da lâmina $=5,40 \pm 3,65$; largura da lâmina $=1,15 \pm$ 0,46 ; comprimento do pedúnculo $=4,46 \pm 2,17$.

DISTRIBUIÇÃO. Oeste da Africa, em águas tropicais; costa paranaense.

MATERIAL EXAMINADO. Rio Guamandituba: 13/7/82, 4 exs. (MCBM-BBI 62); sem data, 12 exs. (MCBM-BBI 63).

DISCUSSÃO. B. bagidaensis é espécie muito semelhante a B. australis, sendo que alguns dos caracteres morfológicos que as distinguem são de difícil observação. Os exemplares examinados diferenciam-se de B. australis por não apresentar serrilhados duplos e pelo fato de a porção calcárea não se estender por dentro dos serrilhados. TURNER (in litt., 1982) discute a possibilidade de uma eventual identidade das duas formas. Apesar de ocorrência reconhecida para a costa oeste da Africa, B. bagidaensis foi referida por TURNER (in litt., 1982) como espécie indo-pacífica. Os exemplares foram encontrados 
em troncos de Rhizophora mangle, juntamente com Bankia rochi. A espécie é registrada pela primeira vez para a costa sul-brasileira.

\section{AGRADECIMENTOS}

Agradecemos à Dr. Ruth Turner pela boa vontade dispensada na confirmação da identificação das espécies. Ao CNPq e à CIRM, pelo auxílio financeiro dado à pesquisa.

\section{REFERENCIAS BIBLIOGRAFICAS}

BARTSCH, P. 1908. A new shipowm from the United States. Proc. Biol. Soc. Washington, 21(34):211-212.

BARTSCH, P. 1920. A new shipworm. Proc. Biol. Soc. Washington, 33(13): 69-70.

BARTSCH, P. 1921. A new classification of the shipworms and description of some new wood boring mollusks. Proc. Biol. Soc. Washington, 34(3): 25-32.

BARTSCH, P. 1922. Monograph of the american shipworms. Bull. U.S. Natl. Mus., 122:1-48.

BARTSCH, P. 1923. Additions to our knowledge of shipworms. Procf. Biol. Soc. Washington, 36:95-102.

BARTSCH, P. 1927. The shipworms of the Phillippine Islands. Bull. U.S. Nat. Mus., 100(2):533-562.

BARTSCH, P. 1944. A new shipworm from Panama canal. Smithson. Misc. Coll., 104(8):1-3.

BASTIDA, R. \& TORTI, M.R. 1972. Organismos perforantes de las costas argentinas. I. La presencia de Lyrodus pedicellatus (Quatrefages, 1849) (Mollusca, Pelecypoda) en el puerto de Mar del Plata. Clave para el reconocimiento de los Teredinidze sudamericanos. Physis, 31(82):39-50.

BOFFI, A.V. 1979. Moluscos brasileiros de interesse médico e econômico. São Paulo, HCITEC. 182. p.

CALLOWAY, C.B. \& TURNEF, R.D. 1983. Documentation and implications of rapid sucessive gametogenic cycles and broods in the shipworm Lyrodus floridanus (Bartsch) (Bivalvia:Teredinidae). Journal of Shelfish Research, 3(1):65-69.

CLAPP, W.F. 1923. A new species of Teredo from Florida (Teredo bartschi). Proc. Bost. Soc. Nat. Hist. 37(2):31-38.

CLAPP, W.F. 1924. Three species of Teredo. Trans. Acad. Sci. St. Louis, 25(1):1-6.

CLENCH, W.J. \& TURNER, R.D. (1946). The genus Bankia in the Western Atlantic. Johnsonia, 2(19):1-28. 
EDMONDSON, C.H. 1942. Teredinidae in Hawaii. Occ. Pap. Bernice P. Bishop Mus., 17(10):97-150.

FERNANDES, L.M.B. \& COSTA, A.F. 1967. Notas sobre organismos marinhos incrustantes e perfurantes das embarcações. B. Est. Pesca, 7(3):8-26.

FORBES, E. \& HANLEY, S. 1835. A history of British Mollusca and their shells. London, John van Voorst. v. I, $486 \mathrm{p}$.

JEFFREYS, J.F. 1860. A synoptical list of the British species of Teredo, with a notice of the exotic species. Ann. Mag. Nat. Hist., 6(3):121-127.

LAMPARELLI, C.C. \& TIAGO, C.G. 1983. Moluscos perfuradores de madeira no litoral de São Paulo; dados preliminares. In: CONGRESSO DE ZOOLOGIA, 10. Resumos. p. 56-57.

LANGE DE MOFRETES, F. 1949. Ensaio de catálogo de moluscos do Brasil. Arq. Mus. Paraná, 7:5-194.

LINNAEUS, C. 1758. Systema naturae per regna tria nature, secundum classes, ordines, genera, species, cum caracteribus, differentiis, synonymis locis. 1. ed. Holminae, v. 1, 824 p.

MANYAK, D.M. 1982. A device for collecting and study of wood boring molluscs; application to boring rates and boring movements of the shipworm Bankia gouldi. Estuaries, 5(5):224-229.

MOLL, F. \& ROCH, F. 1931. The Teredinidae of the British Museum the Natural History Museums at Glasgow and Manchester and the Jeffreys collection. Proc. Malacol. Soc. Lond., 19:201-218, pls. 22-25, test. fig. $1-48$.

MOLL, F. 1935. Über eiige neue Teredinideharten. Sitzungsber. Akad. Wiss. Wien, 144:263-279.

MOLL, 1941. Ubersiaht über dic Terediniden der Museums für Naturkund zu Berlin Sitzungsber. Ges. Naturf. Fr. Berlin:152-225.

MULLER, A.C. 1983. Recrutamento de espécies de Terediindae da baía de Paranaguá (PR). In: CONGRESSO BRASILEIRO DE ZOOLOGIA, 10. Resumos. p.58-59.

RAJOGOPALAIENGAR, R. 1961. A new species of marine borer, Bankia (Neobankia) roonwali (Mollusca: Teredinidae) from India. Science and Culture, 27:550.

RAYNER, S.M. 1979. Comparison of the salinity range tolerance by teredinids (Mollusca:Teredinidae) under controlled conditions with that observed in an estuary in Papua, New Guinea. Aust. J. Mar. Freshw. Res., 30:521-533.

RIMMER, M.A.; BATTAGLENE, S.L.; DOSTINE, P.L. 1983. Observation of Bankia australis Calman (Mollusca:Teredinidae) in the Patonga Creeck mangrove swamp, New South Wales. Aust. J. Mar. Freshw. Res., 30(2): 355-357.

F.IOS, E.C. 1975. Brazilian marine mollusks iconography. Rio Grande, Fundação Universidade do Rio Grande. 331 p. 
ROCH, F. 1931. Die Teredinichen der skandinawichen Museumssammlugen (Stockholm, Gothenburg, Kopenhagen, Oslo, Nidaros und Troms). Ark. Zool., 22A(13):1-29.

ROCH, F. 1955. Die Terediniden ost-und Westindiens der Holländischen Museums-Sammlungen zu Amsterdan und Leiden. Zool. Meded. Rijksmus. Natuur. Hist., Leiden, 34(8):125-151.

ROCH, F. \& MOLL, F. 1929. Die Terediniden der Museum zu Berlin und Hamburg. Mitt. Zool. Staatsinst. Zool. Mus. Hamburg, 44:1-22

ROCH, F. \& MOLL, F. 1935. Ueber einige neue Teredinidenarten. Sitzungsber. Akad. Wiss. Wien. Math. naturw. Klasse Abt., 1,114(5-6):263-269.

SERPA, F.G. 1978. Resistência natural de oito espécies de madeira do Norte e Nordeste do Brasil aos xilófagos marinhos. Curitiba. 60 p. Tese, Mestrado, Universidade Federal do Paraná.

SILVA, S.H.J.; NUNES, A.J.B.; ALVES, M.C.S.; LAJE, V.A. 1980. Contribuição ao estudo das comunidades incrustantes que ocorrem na baía da Guanabará, Rio de Janeiro, Brasil; resultados preliminares. Rev. Bras. Biol., 40(2):367-382.

SILVA, S.H.G.; MARTINS SILVA, M.J.; LAJE, W.; NUNES, A.B. 1983. Organismos marinhos perfurentes de madeira da região de Angra dos Reis. In: CONGRESSO BRASILEIRO DE ZOOLOGIA, 10. Resumos. p. 59-60.

STILLNER, F.J. \& PEDROSO, O. 1977. Verificação da atividade de moluscos do gênero Teredo no litoral oceânico do Rio Grande do Sul. Roessleria, 1(1):131-142.

TURNER, Fi.D. 1966. A survey and illustrated catalogue of the Teredinidae. Cambridge, Mus. of Comp. Zool. Harvard University. 265 p.

TURNER, R.D. 1971. Identification of marine wood-boring molluscs. In: JONES, E.B.G. \& ELTRINGHAM, S.K. Marine borers, fungi and fouling organisms of wood. Paris, Organization for Economic Co-operation and Development. p. 18-63.

TURNER, R.D. \& JOHNSON, A.C. 1971. Biology of marine wood boring molluscs. In: JONES, E.B.G. \& ELTRINGHAM, S.K. Marine borers, fungi and fouling organisms of wood. Paris, Organization for Economic Cooperation and Development. p. 259-296. 


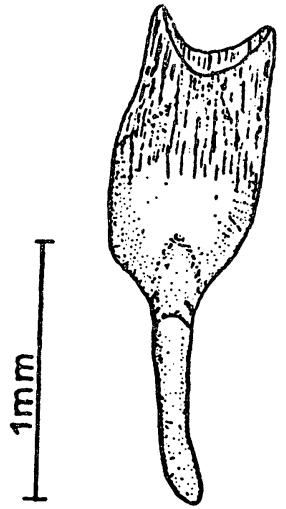

FIG.1

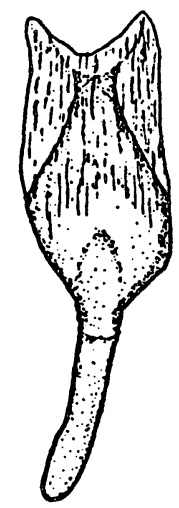

FIG. 2

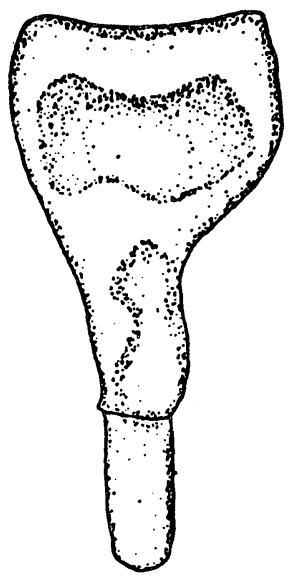

FIG.4

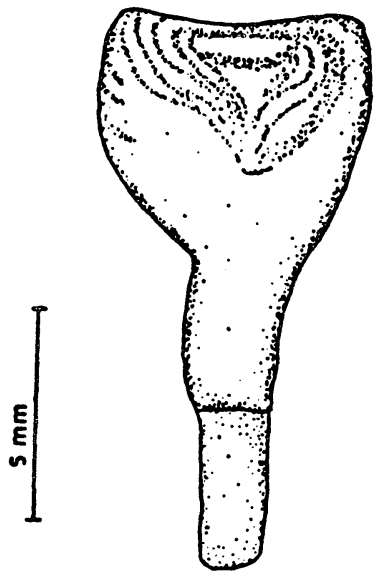

FIG. 3

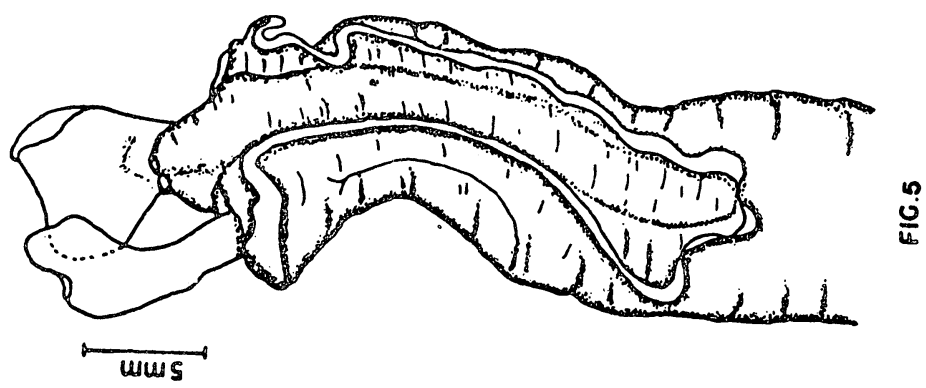

Fig. 1. Lyrodus floridanus. Face externa da paleta.

Fig. 2. Lyrodus floridanus. Face interna da paleta.

Fig. 3. Neoteredo reynei. Parte posterior do animal.

Fig. 4. Neoteredo reynei. Face externa da paleta.

Fig. 5. Neoteredo reynei. Face interna da paleta. 


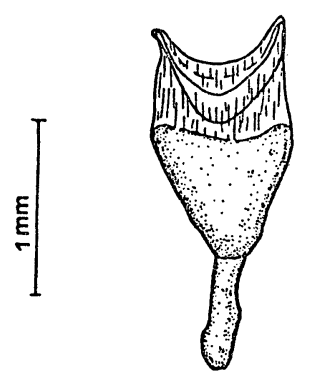

FIG.6

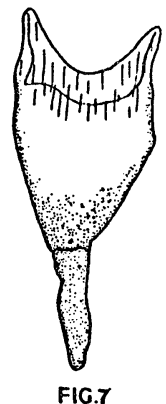

FIG.7

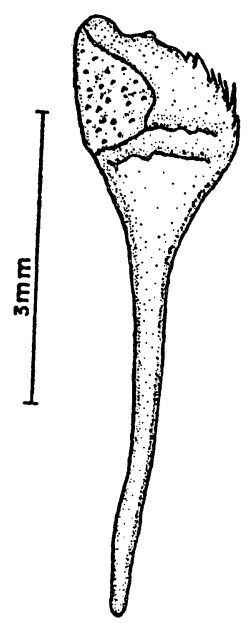

FIC.12

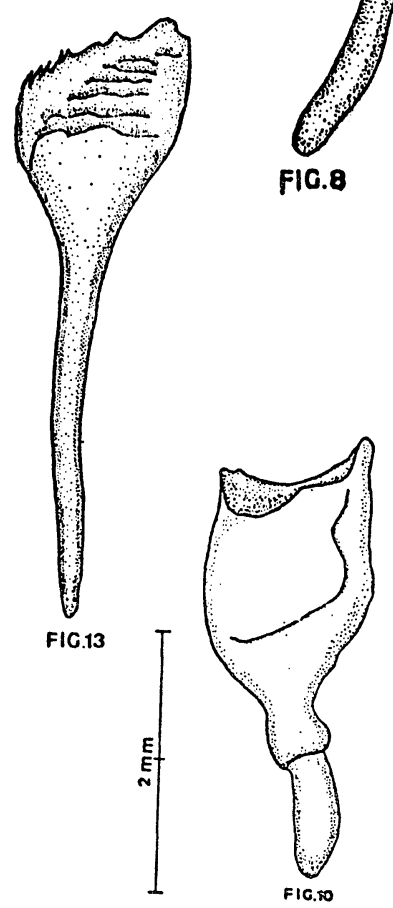

Fig. 6. Teredo navalis. Face externa da paleta.

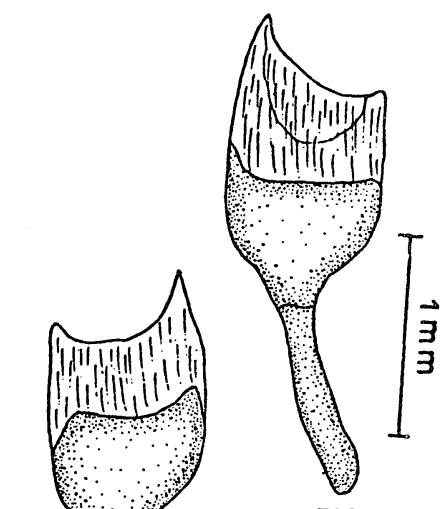

FIG.9

Fig. 7. Teredo navalis. Face interna da paleta.

Fig. 8. Teredo bartschi. Face interna da paleta.

Fig. 9. Teredo bartschi. Face externa da paleta.

Fig. 10. Teredo mindanensis. Face interna da paleta.

Fig. 11. Teredo mindanensis. Face externa da paleta.

Fig. 12. Nausitora fusticula. Face externa da paleta.

Fig. 13. Nausitora fusticula. Face interna da paleta. 

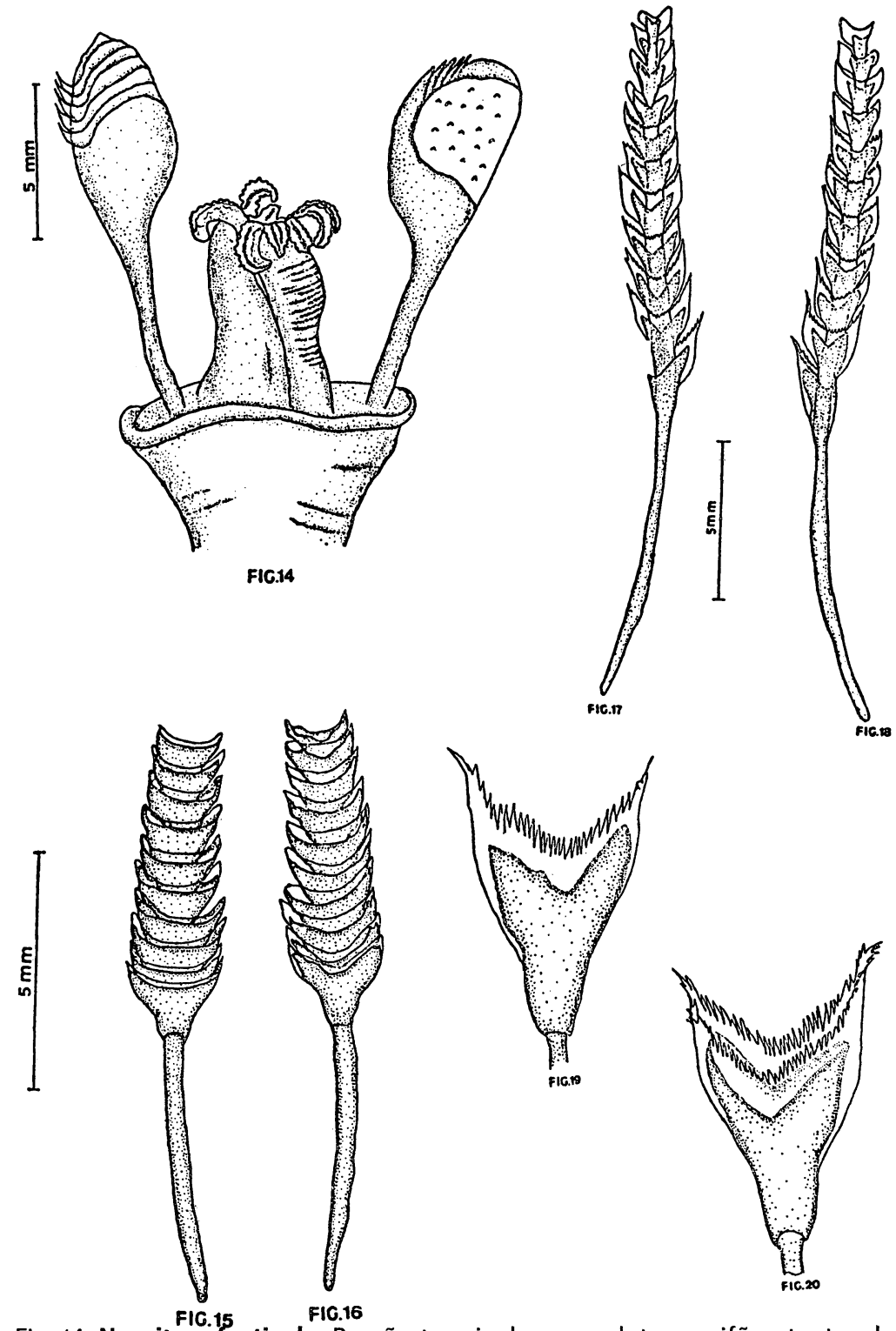

Fig. 14. Nausitora fusticula. Porção terminal, com paletas e sifões tentaculados.

Fig. 15. Bankia gouldi. Face externa da paleta.

Fig. 16. Bankia gouldi. Face interna da paleta.

Fig. 17. Bankia fimbriatula. Face externa da paleta.

Fig. 18. Bankia fimbriatula. Face interna da paleta.

Fig. 19. Bankia fimbriatula. Face externa de um cone da paleta.

Fig. 20. Bankia fimbriatula. Face interna de um cone da paleta. 

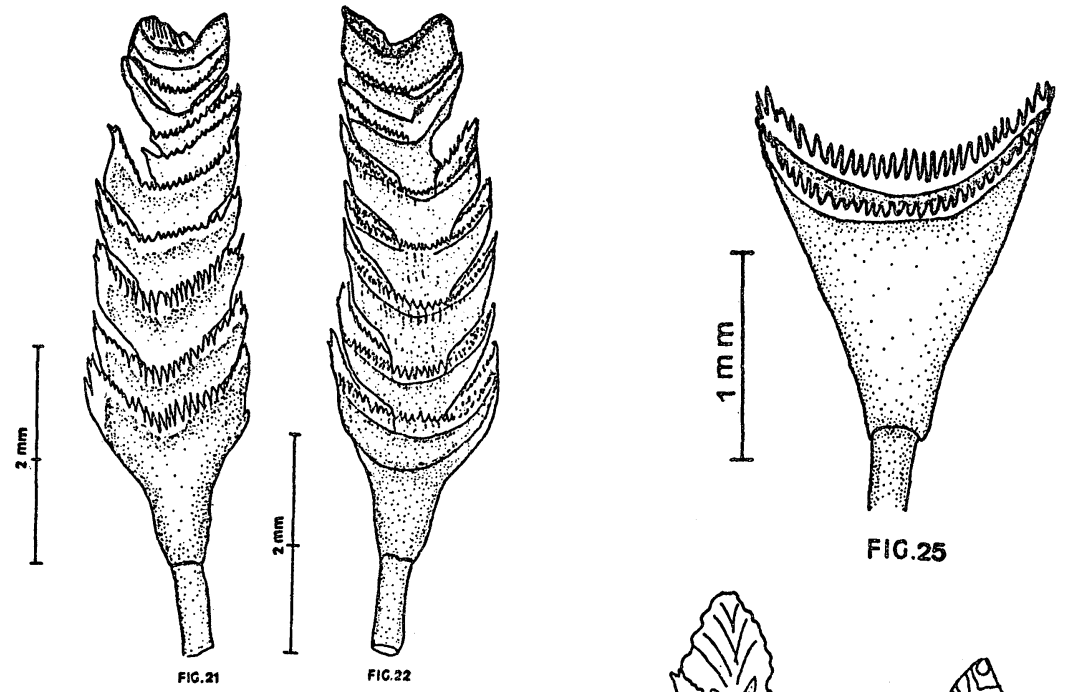

FIG.25
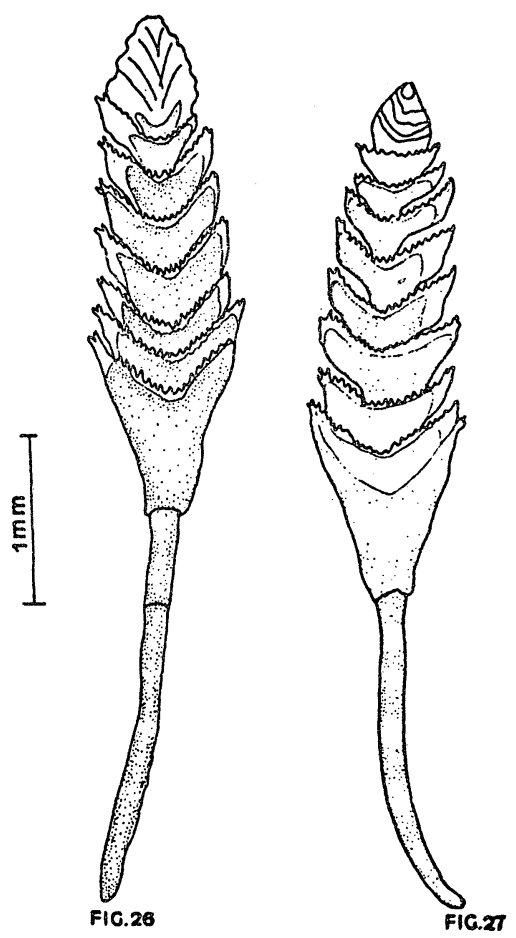

Fig. 21. Bankia rochi. Face externa da paleta.

Fig. 22. Bankia rochi. Face interna da paleta.

Fig. 23. Bankia rochi. Face interna de dois segmentos da paleta.

Fig. 24. Bankia rochi. Face externa de dois segmentos da paleta.

Fig. 25. Bankia bagidaensis. Face externa de um cone individual da paleta.

Fig. 26. Bankia bagidaensis. Face interna da paleta.

Fig. 27. Bankia bagidaensis. Face externa da paleta. 


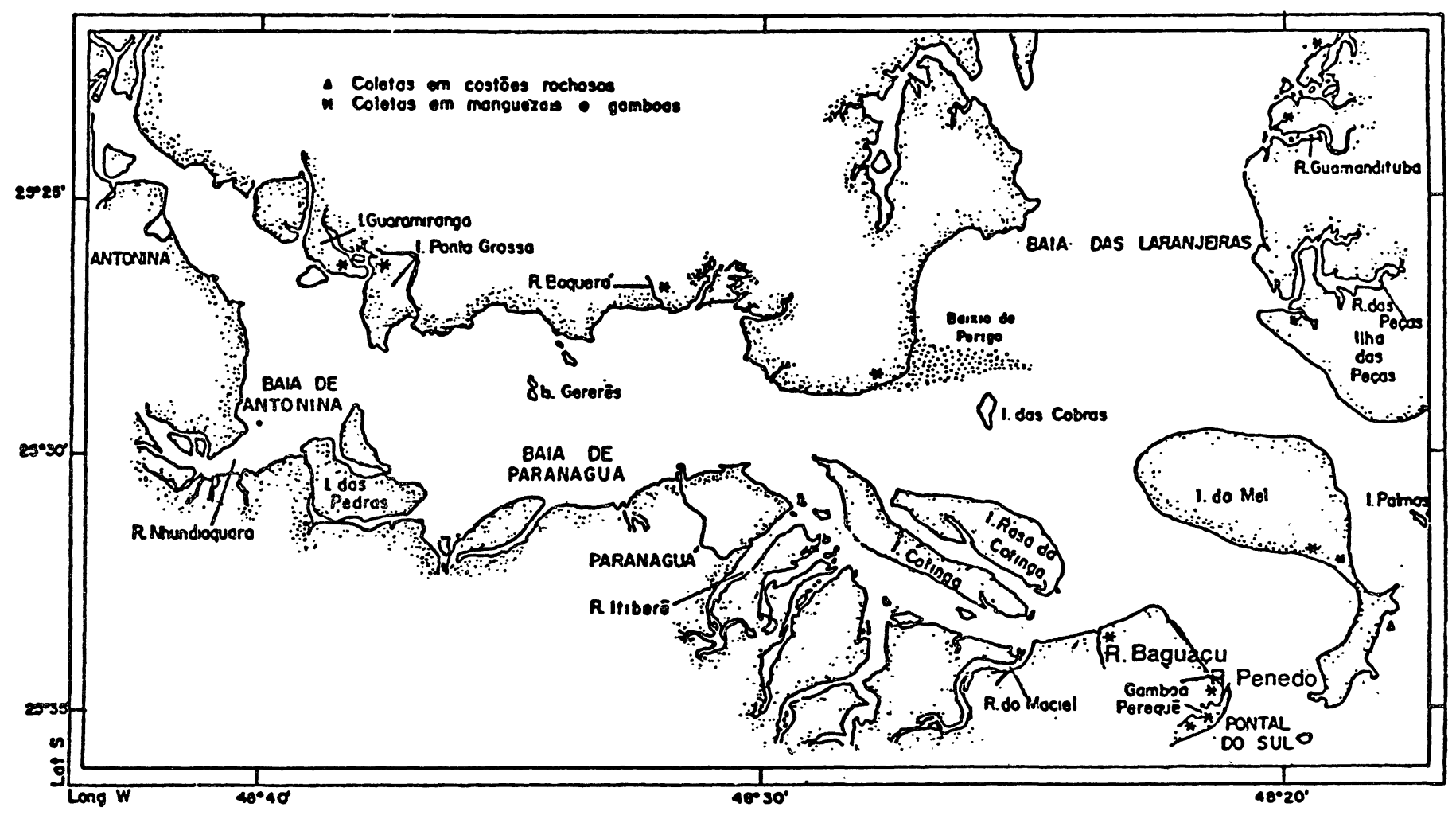

Mapa 1 - Mapa da Baía de Paranaguá com posicionamento das estações de coleta 


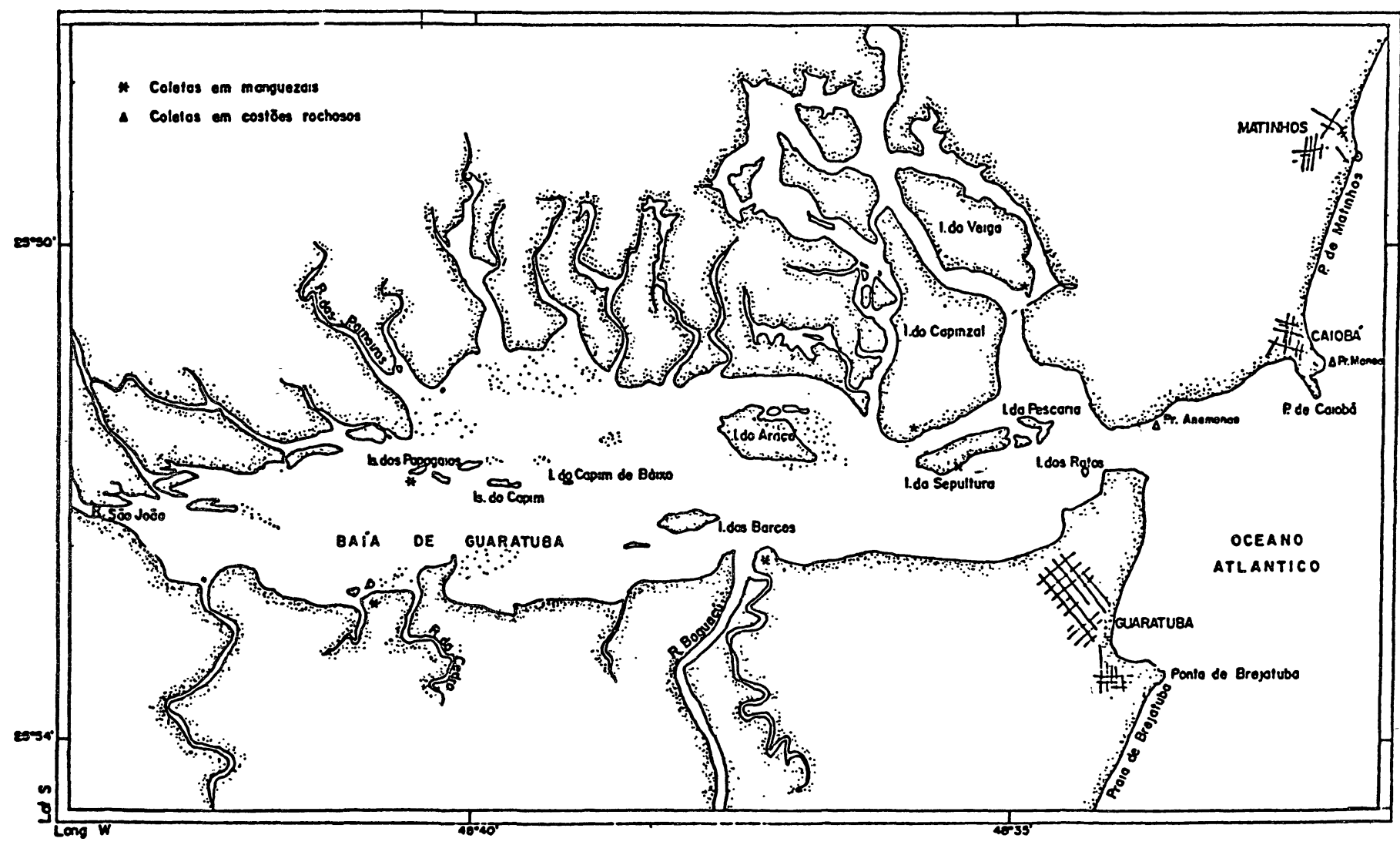

Mapa 2 - Mapa da Baía de Guaratuba e adjacências, com posicionamento dos pontos e estações de coleta 